\title{
Control of the electromagnetic field in a cavity by an external perturbation
}

\author{
Michele Cotrufo $^{\mathrm{a}}$, Ewold Verhagen ${ }^{\mathrm{b}}$, and Andrea Fiore ${ }^{\mathrm{a}}$ \\ ${ }^{a}$ Department of Applied Physics, Eindhoven University of Technology, 5600 MB Eindhoven, \\ The Netherlands \\ ${ }^{\mathrm{b}}$ Center for Nanophotonics, FOM Institute AMOLF, Science Park 104, 1098 XG Amsterdam, \\ The Netherlands
}

\begin{abstract}
A prototypical experiment in cavity quantum electrodynamics involves controlling the light-matter interaction by tuning the frequency of a cavity mode in- and out-of resonance with the frequency of a quantum emitter, ${ }^{1-3}$ while the field amplitude is generally unaltered. The opposite situation, where one perturbs the spatial pattern of a cavity mode without changing its frequency, has been considered only recently in a few works. ${ }^{4,5}$ Changing the amplitude of the field at the emitter's position has important applications, at it allows a real-time control of the light-matter coupling rate, and therefore a direct control of processes such as spontaneous emission and Rabi oscillations. In view of this large potential, in this paper we discuss general design principles that allow obtaining large variations of the electromagnetic field, without change of the frequency, upon an external perturbation of the cavity. We showcase the application of these rules to two photonic structures, a single Fabry-Perot cavity and a coupled three-cavity system. As showed by our analysis and by the examples provided, a small frequency spacing between the modes of the unperturbed cavity is an important requirement to obtain large field variations upon small perturbations. In this regard, a coupled-cavity system, where the frequency spacing is controlled by the interaction rates between the single cavities, constitutes the most promising system to achieve large modulations of the field amplitude.
\end{abstract}

Keywords: cavity QED, optomechanics, field variation, field control, perturbation theory, coupled cavity

\section{INTRODUCTION}

Controlling the modes of an optical cavity is crucial for cavity quantum electrodynamics and light-matter interaction experiments. In many situations, a reversible and post-fabrication tuning of the frequency of a cavity mode is required in order to change the spectral overlap between the mode and a quantum emitter and, therefore, to control the light-matter interaction. ${ }^{1-3}$ Such tuning can in principle be obtained by changing the refractive index of the cavity material by, e.g., thermal effects ${ }^{6,7}$ or carrier-injection effects in semiconductor materials. ${ }^{8}$ Another class of approaches relies on a mechanical perturbation of the cavity structure itself, realized by, e.g., approaching and perturbing the optical near field by an external object, such as a tip,,${ }^{9,10}$ or changing the distance between different parts of the cavity. In this regard, micro and nano opto-electro-mechanical systems have been often used as an elegant and reliable solution to implement spectral control on devices such as vertical cavity surface emitting lasers ${ }^{11}$ and, recently, photonic crystal cavities. ${ }^{3,12-17}$

In all the approaches mentioned above, the external perturbation to the cavity leads to a change of the resonant frequency, while the field spatial distribution is weakly altered. This is desirable whenever one wants to control the light-matter interaction strength only through the spectral detuning between an emitter and the cavity mode, ${ }^{1-3}$ without changing the value of the electric field amplitude at the emitter position. As a diametrically opposite situation, we can consider systems in which an external perturbation induces a change of the spatial pattern of the cavity field, while the resonant frequency is weakly or negligibly affected. The possibility of spatially shaping the field is quite attractive in various contexts. Indeed, the amplitude of the mode field at the emitter's position directly determines the emitter-field coupling rate, $g$, and affects the radiative processes such as spontaneous emission and Rabi oscillations. For example, if the electric field amplitude can be controlled on timescales shorter than $1 / \mathrm{g}$, real-time control of cavity QED processes becomes possible. ${ }^{4,5}$ Additionally, 
modifications of the field profile can lead to a change of the optical quality factor $\mathrm{Q}$ by changing the field intensity in regions with losses, which could have important application in the context of Q-switched lasers. ${ }^{19}$ For the specific case in which the perturbation is created by the displacement of a part of the cavity, a mechanical variation of the $\mathrm{Q}$ factor has important applications in the field of dissipative optomechanics. ${ }^{20}$ Moreover, we have recently shown that a system in which a mechanical resonator affects the cavity field without changing its frequency can be used to establish a direct interaction between a quantum emitter and the resonator. ${ }^{21}$

In this paper we outline the general design principles which allow obtaining, upon external perturbation of an optical cavity, large field variations, without change of the optical frequency. In sec. 2, we analyze the problem with a first-order perturbation theory approach, first in the framework of a generic eigenvalue problem (which therefore applies also to other physical cases of interest, such as the Schrödinger equation), and then for the specific case of the electromagnetic field of an optical cavity. After having identified the minimal requirements that lead to large field variation, in sec. 3 we show numerical calculations related to two realistic photonic structures, namely a Fabry-Perot cavity (sec. 3.1) and a system composed by three coupled photonic crystal cavities (sec. 3.2). This latter system has been recently shown to provide large change of the electric field in the central cavity upon a specific perturbation scheme. ${ }^{5}$ The general principles outlined in this paper allow us to understand what makes these systems, and the particular perturbations used, successful in providing large field variations.

\section{GENERAL THEORY}

The Maxwell equations that determine the frequency and the spatial profile of the electromagnetic field can be cast, for the case of linear, isotropic, and dispersionless dielectric materials, in a eigenvalue problem. ${ }^{22} \mathrm{In}$ particular, assuming a harmonic time-dependence of the fields, the magnetic field is given by the solution of the equation

$$
\hat{\Theta} \mathbf{H}(\mathbf{r})=\left(\frac{\omega}{c}\right)^{2} \mathbf{H}(\mathbf{r})
$$

where $\hat{\Theta}=\nabla \times\left(\frac{1}{\epsilon(\mathbf{r})} \nabla \times\right)$ is a linear Hermitian operator, $\omega$ is the angular frequency (i.e., the eigenvalue), $\mathbf{H}(\mathbf{r})$ is the magnetic field (i.e., the eigenvector) and $\epsilon(\mathbf{r})$ is the dielectric function. Equation 1 allows a more formal explanation of the effects discussed above. Let us assume that the system is weakly perturbed by some mean, which leads to a change of the dielectric function and, therefore, of the operator $\hat{\Theta}$. Under a generic perturbation, both the eigenvalue $\omega$ and the eigenvector $\mathbf{H}(\mathbf{r})$ will change. However, as shown below, in many practical realizations the perturbation is such that it induces a strong change of $\omega$ but a weak or null change of $\mathbf{H}(\mathbf{r})$. Here, we are interested in finding minimal requirements such that the opposite holds: the eigenvalue $\omega$ is not affected while $\mathbf{H}(\mathbf{r})$ is strongly changed. We consider a generic eigenvalue problem, in the form

$$
\hat{\mathcal{H}}_{0} \Psi_{n}^{(0)}(\mathbf{r})=E_{n}^{(0)} \Psi_{n}^{(0)}(\mathbf{r})
$$

where $\hat{\mathcal{H}}_{0}$ is a linear Hermitian operator which acts on complex functions defined on a domain $D$. We assume that appropriate conditions are imposed on $D$ and its boundaries, so that the spectrum of the eigenvalues is discrete. For simplicity, we also assume that the spectrum is not degenerate. The solutions of the problem in eq. 2 are then given by a set of eigenvalues $E_{n}^{(0)}$ and eigenfunctions $\Psi_{n}^{(0)}(\mathbf{r})$ labelled by $n$, and arranged such that $E_{n}^{(0)}<E_{n+1}^{(0)}$. The superscripts (0) indicate that these quantities are associated with the unperturbed system. We will refer to the eigenvalues and eigenfunctions as energies and fields, respectively, for brevity. Furthermore, we assume that the operator $\hat{\mathcal{H}}_{0}$ has an inversion symmetry with respect to a plane, e.g., the plane $y z$. In this case all the fields $\Psi_{n}^{(0)}(\mathbf{r})$ will be either symmetric or antisymmetric with respect to the inversion $x \rightarrow-x$. In the following, when using the terms symmetric and anti-symmetric, we will always refer to this particular transformation.

We now introduce a small perturbation to the system, i.e., $\hat{\mathcal{H}}=\hat{\mathcal{H}}_{0}+\hat{\mathcal{V}}$. The energies and fields of the operator $\hat{\mathcal{H}}$ are obtainable by perturbative expansions in which the zero-order terms are the unperturbed energies and fields,

$$
E_{n}=E_{n}^{(0)}+E_{n}^{(1)}+E_{n}^{(2)}+\ldots
$$




$$
\Psi_{n}(\mathbf{r})=\Psi_{n}^{(0)}(\mathbf{r})+\Psi_{n}^{(1)}(\mathbf{r})+\Psi_{n}^{(2)}(\mathbf{r})+\ldots .
$$

The standard non-degenerate perturbation theory allows calculating the first-order corrections,

$$
\begin{gathered}
E_{n}^{(1)}=\int d \mathbf{r} \Psi_{n}^{(0) *}(\mathbf{r}) \hat{\mathcal{V}} \Psi_{n}^{(0)}(\mathbf{r})=\left\langle\Psi_{n}^{(0)}|\hat{\mathcal{V}}| \Psi_{n}^{(0)}\right\rangle \\
\Psi_{n}^{(1)}(\mathbf{r})=\sum_{k \neq n} \frac{\int d \mathbf{r} \Psi_{k}^{(0) *}(\mathbf{r}) \hat{\mathcal{V}} \Psi_{n}^{(0)}(\mathbf{r})}{E_{n}^{(0)}-E_{k}^{(0)}} \Psi_{k}^{(0)}(\mathbf{r})=\sum_{k \neq n} \frac{\left\langle\Psi_{k}^{(0)}|\hat{\mathcal{V}}| \Psi_{n}^{(0)}\right\rangle}{E_{n}^{(0)}-E_{k}^{(0)}}\left|\Psi_{k}^{(0)}\right\rangle
\end{gathered}
$$

where we have expressed the results also in the bra-ket notation (with abuse of notation, we use the same symbol $\hat{\mathcal{V}}$ for the operator acting on the functions space and the operator acting on the abstract space). We now consider the following question: Which property should the operator $\hat{\mathcal{V}}$ have such that, at the first perturbative order, the energy is not changed (i.e., $E_{n}^{(1)}=0$ ) while the correction $\Psi_{n}^{(1)}(\mathbf{r})$ to the field pattern is 'large'?

A sufficient condition to obtain $E_{n}^{(1)}=\left\langle\Psi_{n}^{(0)}|\hat{\mathcal{V}}| \Psi_{n}^{(0)}\right\rangle=0$ is that the states $\left|\Psi_{n}^{(0)}\right\rangle$ and $\hat{\mathcal{V}}\left|\Psi_{n}^{(0)}\right\rangle$ have opposite symmetry, which means that the operator $\mathcal{V}$ must be anti-symmetric. The same condition can also lead to large field variations at the first perturbative order. Indeed, large terms in the sum of eq. 6 appear when, for closely spaced unperturbed energies (i.e., $\left|E_{n}^{(0)}-E_{k}^{(0)}\right| \approx 0$ ), the numerator $\left\langle\Psi_{k}^{(0)}|\hat{\mathcal{V}}| \Psi_{n}^{(0)}\right\rangle$ is not zero. We can therefore obtain large corrections at the first perturbative order if, for the terms corresponding to the energetically closest states $(k=n \pm 1)$, the corresponding matrix elements do not vanish, i.e., $\left\langle\Psi_{n \pm 1}^{(0)}|\hat{\mathcal{V}}| \Psi_{n}^{(0)}\right\rangle \neq 0$.

For many uni-dimensional operators $\hat{\mathcal{H}}_{0}$ of physical interest*, the following oscillation theorem holds: ${ }^{23}$ the symmetry of a state $\Psi_{n}^{(0)}(x)$ is opposite to the ones of the energetically closest states $\Psi_{n+1}^{(0)}(x)$ and $\Psi_{n-1}^{(0)}(x)$ (where we have replaced the three-dimensional coordinate $\mathbf{r}$ by the uni-dimensional coordinate $x$ ). For three-dimensional systems and vectorial fields, we can still use the results of the oscillation theorem, as long as we consider only modes that differ for their oscillation pattern along the $x$-direction, but that have the same symmetry along the other two directions $(y$ and $z$ ). In many practical situations (see examples below) this is possible because the undesired modes are energetically far from the modes of interest. Moreover, even when the undesired modes are energetically close, they often have different field polarizations with respect to the modes of interest, so that they do not contribute to the sum in eq. 6 .

Due to the opposite symmetry of $\left|\Psi_{n}^{(0)}\right\rangle$ and $\left|\Psi_{n \pm 1}^{(0)}\right\rangle$, the requirement $\left\langle\Psi_{n \pm 1}^{(0)}|\hat{\mathcal{V}}| \Psi_{n}^{(0)}\right\rangle \neq 0$ implies again that $\hat{\mathcal{V}}$ must be anti-symmetric. However, this condition is in general not sufficient to guarantee large field variations at the first perturbative order, as discussed below. We also note that similar arguments imply that, at the first perturbative order, large energy variations and small field variations are instead obtained if $\hat{\mathcal{V}}$ is symmetric. This explains why this situation is the most common: a uniform perturbation applied to the entire structure, such as a change of the refractive index by heating, is symmetric.

In order to derive a semi-quantitative formula for the field variation in a particular point $\overline{\mathbf{r}}$, we consider only the coupling between the nearest states $(k=n \pm 1)$, and we assume that the energy spacings are equal (i.e., $\left.\mathcal{E} \equiv E_{n+1}^{(0)}-E_{n}^{(0)} \approx E_{n}^{(0)}-E_{n-1}^{(0)}\right)$. Moreover, we assume that, in the point $\overline{\mathbf{r}}$ of interest, the fields $\Psi_{n-1}^{(0)}$ and $\Psi_{n+1}^{(0)}$ have approximately the same amplitude $\left(\left|\Psi_{n-1}^{(0)}(\overline{\mathbf{r}})\right| \approx\left|\Psi_{n+1}^{(0)}(\overline{\mathbf{r}})\right|\right)$. As will be shown below, this is indeed a common situation in realistic photonic structures. Since the phase difference between eigenstates is arbitrary, we chose it such that $\Psi_{n-1}^{(0)}(\overline{\mathbf{r}}) \approx \Psi_{n+1}^{(0)}(\overline{\mathbf{r}})$. The first-order variation of the field $\Psi_{n}^{(0)}$ at the point $\overline{\mathbf{r}}$ is

$$
\Psi_{n}^{(1)}(\overline{\mathbf{r}}) \approx \frac{\Psi_{n \pm 1}^{(0)}(\overline{\mathbf{r}})}{\mathcal{E}}\left[\left(\left\langle\Psi_{n-1}^{(0)}\left|-\left\langle\Psi_{n+1}^{(0)}\right|\right) \hat{\mathcal{V}} \mid \Psi_{n}^{(0)}\right\rangle\right]\right.
$$

In order to obtain large variations of the field $\Psi_{n}^{(0)}$ at the point $\overline{\mathbf{r}}$ we therefore need that (I) the frequency spacing between the modes, $\mathcal{E}$, is small, (II) the fields $\Psi_{n \pm 1}^{(0)}$ have large values at $\overline{\mathbf{r}}$, and (III) the matrix elements

\footnotetext{
* More specifically, for operators that are linear combinations of derivative operators up to the second order and multiplication by a function, like, i.e., the Hamiltonian of a quantum particle in a 1D potential, or the operator of the 1D Helmoltz equation. The eigenvalue equations obtained with these operators are both particular cases of the Sturm-Liouville problem, for which the oscillation theorem holds. ${ }^{23}$
} 
$\left\langle\Psi_{n-1}^{(0)}|\hat{\mathcal{V}}| \Psi_{n}^{(0)}\right\rangle$ and $\left\langle\Psi_{n+1}^{(0)}|\hat{\mathcal{V}}| \Psi_{n}^{(0)}\right\rangle$ have opposite sign. The condition (III) is satisfied when, in the regions where the perturbation is large, the fields $\Psi_{n-1}^{(0)}(\mathbf{r})$ and $\Psi_{n+1}^{(0)}(\mathbf{r})$ have opposite sign. We note that the phase of the fields (and therefore their relative sign in every point) is fixed by the requirement $\Psi_{n-1}^{(0)}(\overline{\mathbf{r}}) \approx \Psi_{n+1}^{(0)}(\overline{\mathbf{r}})$. Because of the opposite symmetries of $\Psi_{n}^{(0)}$ and $\Psi_{n \pm 1}^{(0)}$, the requirement (II) suggests that large field variations will be obtained at points $\overline{\mathbf{r}}$ where $\Psi_{n}^{(0)}(\overline{\mathbf{r}})=0$.

\section{EXAMPLES}

We now apply the principles outlined above to realistic photonic nanostructures. Perturbations are implemented through local changes of the refractive index. In both examples we assume that the external perturbation can locally increase or decrease the refractive index. This can be readily obtained in practice by heating the systems (or injecting free carriers) with, e.g., different laser beams impinging in different areas and with the same power, such that a uniform increase of the refractive index is initially obtained. Then, by reducing the power of certain beams, an effective reduction of the refractive index is obtained in specific areas (with respect to the other parts of the cavity). All the calculations are performed with a commercial Finite Element Method solver (COMSOL Multiphysics).

\subsection{Fabry-Perot cavity}

We first consider the case of a rectangular cavity made of a material with refractive index $\mathrm{n}=3.4$ and surrounded by perfectly reflecting mirror (fig. 1a). The dimensions of the cavity are $L=2.5 \mu m(\mathrm{x}) \times 0.21 \mu \mathrm{m}(\mathrm{y}) \times 0.23$ $\mu m(\mathrm{z})$, and they are chosen such that the four lowest frequency modes are $y$-polarized and oscillate along the $\mathrm{x}$-direction. Moreover, these dimensions ensure that no degenerate modes are present in the lowest part of the spectrum. The refractive index is perturbed along the $x$-direction in either a symmetric $(\delta n(x)=\Delta n \cdot \cos (\pi x / L)$, fig. 1) or anti-symmetric $(\delta n(x)=\Delta n \cdot \sin (2 \pi x / L)$, fig. 2) way. In fig. 1c (middle row), we show the spatial pattern of the first four modes in an $x z$-plane in the middle of the cavity. As expected, the fields feature an increasing number of lobes along the $x$-direction, and they have alternating symmetry. When the symmetric perturbation (fig. 1b) is added, no appreciable change of the mode patterns is visible (upper and lower rows of fig. 1c). The only effect is a weak shrinking or enlarging of the lobes, for $\Delta n<0$ and $\Delta n>0$ respectively. The value of the fields in a fixed point (e.g., $\mathbf{r}=0$, fig. 1e) is almost unaffected by the perturbation. On the contrary, the field frequencies (fig. 1d) evolve linearly with $\Delta n$.

When the refractive index is instead perturbed in an anti-symmetric way (fig. 2b), the opposite situation is observed. The mode frequencies (fig. 2d) are all constant for small values of $\Delta n$, as the perturbative corrections are of the second or higher order. The field patterns are instead strongly affected by the perturbation (see plots in fig. 2c), in particular for the first two modes. In fig. 2e we plot the values of the four fields at $\mathbf{r}=0$ versus $\Delta n$. For the second and fourth mode (green and cyan line, respectively), a linear variation of the field amplitude is obtained for small perturbations. The first and third mode (blue and red line, respectively) show instead a quadratic behaviour. This is due do the fact that the nearest unperturbed modes to which they can couple (the second and the fourth one) have zero amplitude at $\mathbf{r}=0$, so that the first-order field perturbation (eq. 7 ) is zero. The fact that the fourth mode is less affected than the second one (compare the slope of the green and cyan lines in fig. 2e) is due to the uneven frequency spacing between the unperturbed modes (see fig. $2 \mathrm{~d}$ for $\Delta n=0$ ), as discussed above (see eqs. 6 and 7).

\subsection{Three coupled L3 cavities}

The example provided in the previous section showed that large field variations can be obtained with a simple optical cavity. However, the system is affected by an important drawback: the frequency spacing between the modes is dictated by the cavity dimensions, and it is of the same order of magnitude of the frequency of the first mode. As explained above, the first-order variation of the field patterns is inversely proportional to the frequency spacing between the modes (eq. 7), and therefore small spacings are required to have large effects. For the case of a single cavity, any attempt to reduce the frequency spacing will also reduce the mode frequencies, thus changing the spectral range in which the cavity operates. 

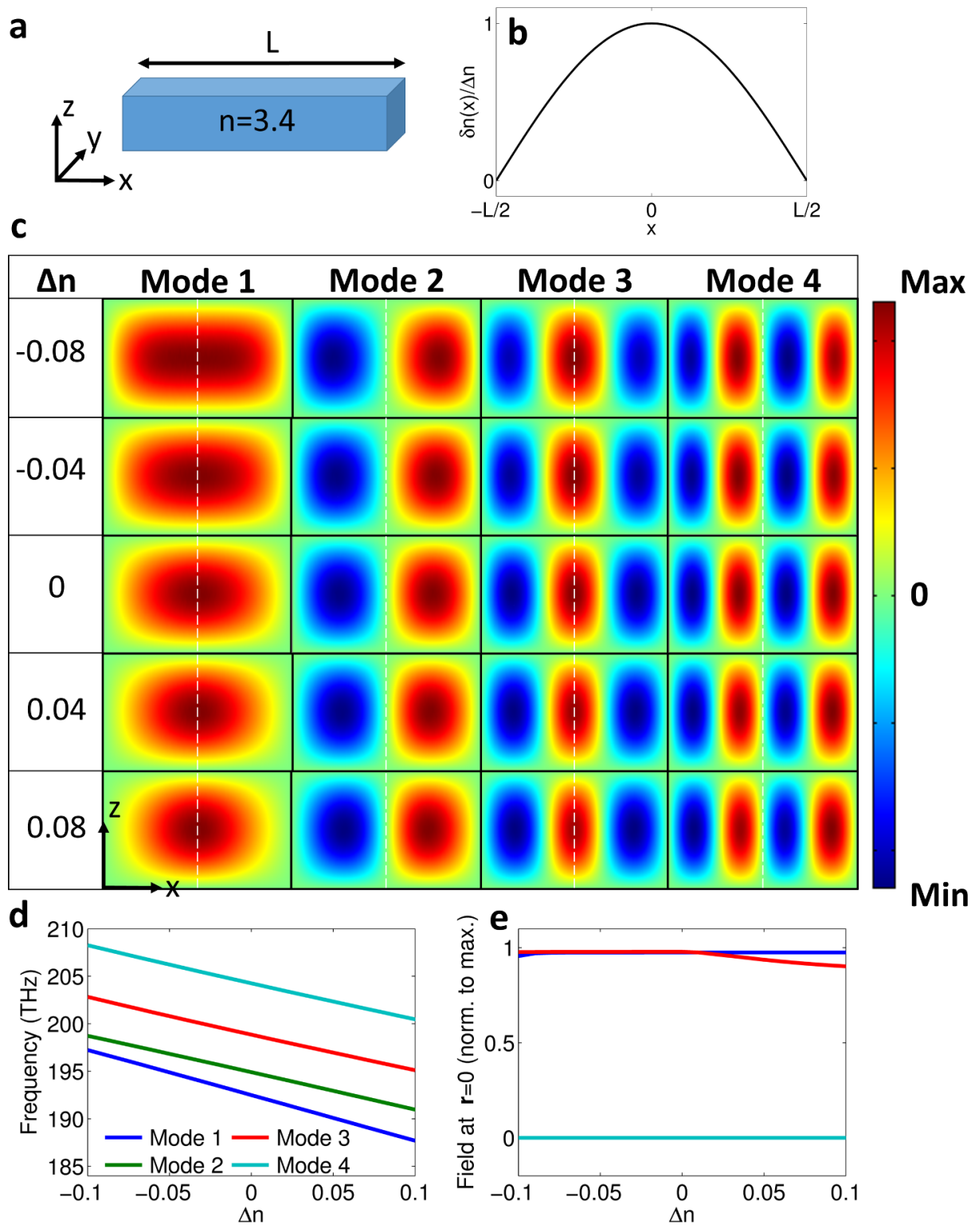

Figure 1. Modes of a rectangular cavity (panel a) upon a symmetric perturbation of the refractive index. The perturbation (panel b) is $\delta n(x)=\Delta n \cdot \cos (\pi x / L)$. The table in panel (c) shows the field profiles $(y$-component of the electric field) of the first four modes for different values of the parameter $\Delta n$, as indicated in the first column. Each mode is normalized to its maximum value. The phases of the modes 1 and 3 is chosen such that they have the same sign at $\mathrm{r}=0$ for $\Delta n=0$. The vertical dashed lines mark the $\mathrm{r}=0$ position. (d) Mode frequencies versus $\Delta n$. (e) Values of the four fields at $\mathbf{r}=0$ versus the parameter $\Delta n$ (the curve corresponding to modes 2 and 4 are on top of each other).

This drawback can be circumvented by working with systems of coupled cavities. When two or more identical cavities are placed close to each other, their respective fields will partially overlap. This creates supermodes, i.e., linear combinations of the single cavity modes, with well-defined symmetries. The frequencies of the supermodes are different, and the frequency spacing is dictated by the interaction rate, $J$, between the single-cavity fields. This interaction rate is controlled by the cavity distances and the overlap of the respective fields, ${ }^{24}$ and can be several order of magnitudes smaller than the cavity frequency. This kind of systems is therefore promising in view of reaching small frequency spacings (and therefore large field variations) without affecting the optical mode frequencies. Importantly, if the single cavities are arranged in such a way that the system has an inversion 

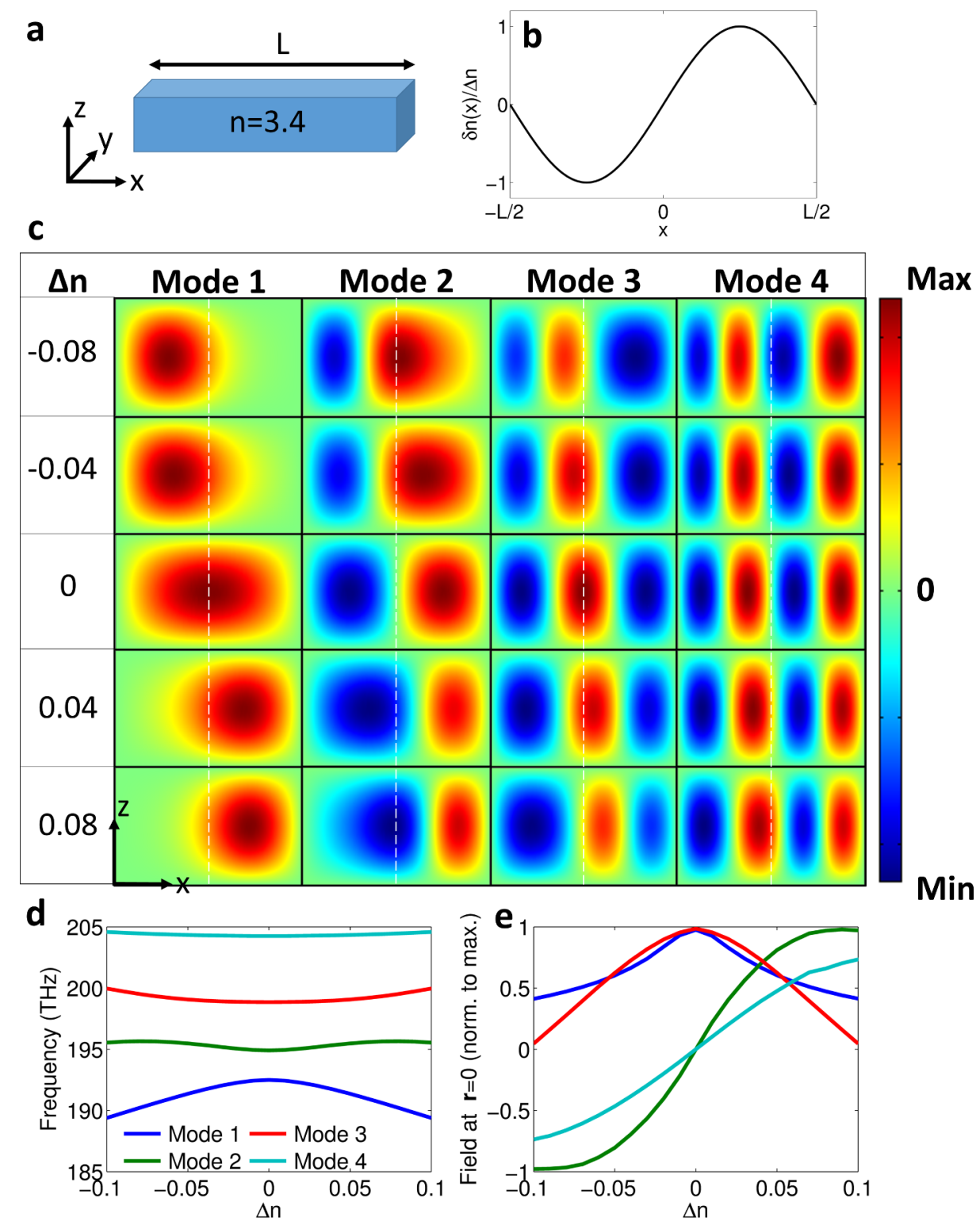

$\mathrm{x}$

Figure 2. Modes of a rectangular cavity (panel a) upon an anti-symmetric perturbation of the refractive index. The perturbation (panel b) is $\delta n(x)=\Delta n \cdot \sin (2 \pi x / L)$. The table in panel (c) shows the field profiles of the first four modes for different values of $\Delta n$, as indicated in the first column. Each mode is normalized to its maximum value. The phases of the modes 1 and 3 is chosen such that they have the same sign at $\mathbf{r}=0$ for $\Delta n=0$. The vertical dashed lines mark the $\mathbf{r}=0$ position. (d) Mode frequencies versus $\Delta n$. (e) Values of the four fields at $\mathbf{r}=0$ versus $\Delta n$ (the curve corresponding to modes 2 and 4 are on top of each other).

symmetry point, a symmetric or anti-symmetric perturbation can be obtained by properly detuning each cavity.

We focus on the particular case of three in-line coupled cavities, ${ }^{5,25,26}$ where the central cavity interacts with the lateral ones, while the interaction between the lateral cavities is negligible. As recently shown, ${ }^{5}$ large variations of the electric field in the central cavity are obtained when the lateral cavities are detuned in an equal and opposite way. This behaviour is precisely the consequence of the principles outlined in sec. 2, as we show here. We consider the case of three L3 photonic crystal $(\mathrm{PhC})$ cavities. Each cavity is realized by removing three collinear holes in a triangular pattern of air holes embedded in a dielectric (fig. 3a). We use a lattice constant $\mathrm{a}=$ 


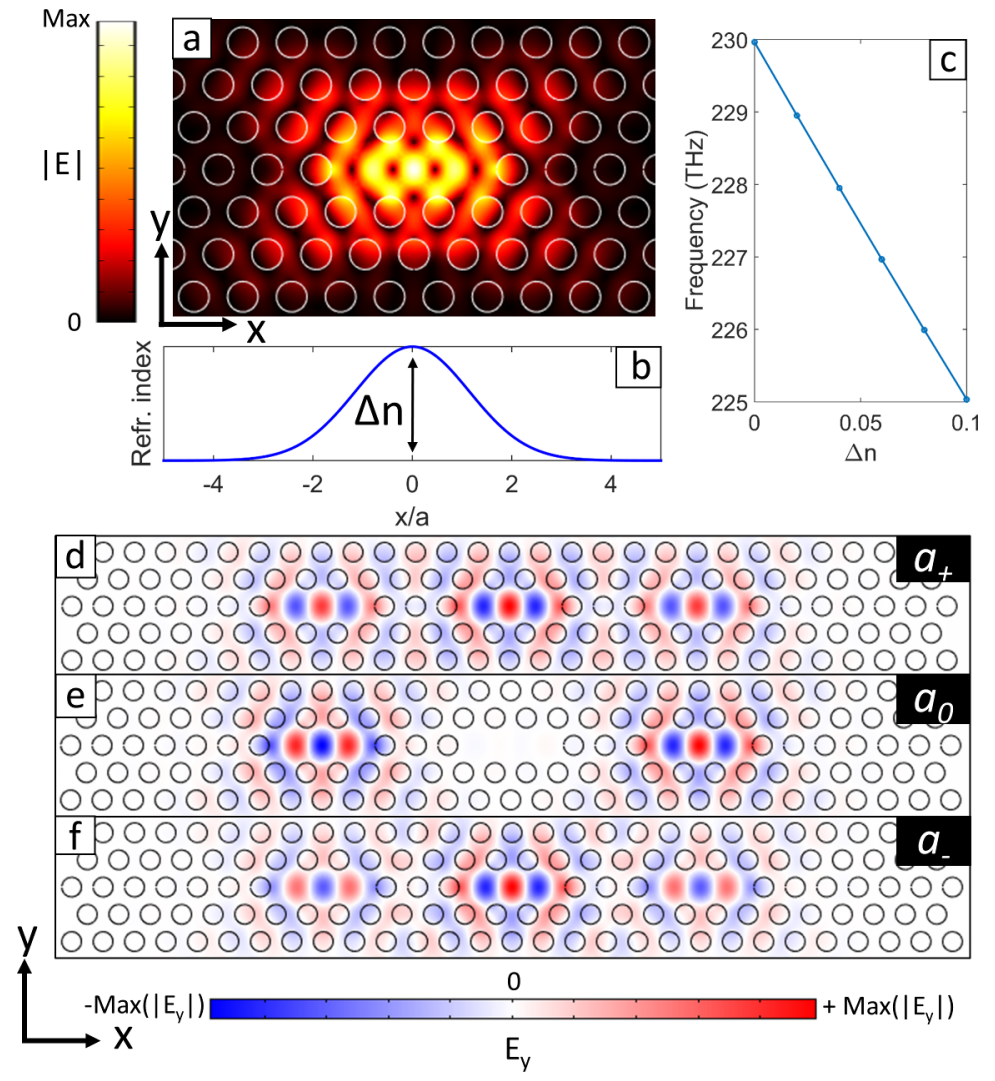

Figure 3. (a-c) A single L3 photonic crystal cavity. The bulk refractive index is locally changed by a gaussian perturbation (panel b) with a width of $600 \mathrm{~nm}$ and a varying amplitude $\Delta n$. The horizontal axis of panel b is in units of the lattice constant a. Panels a and c show, for the fundamental $y$-polarized mode, the field profile (for zero perturbation) and the frequency versus the refractive index modulation $\Delta n$. (d-f) Field patterns ( $y$ component of the electric field) of the three optical supermodes that are formed when three L3 cavities are coupled in-line and separated by two periods.

$370 \mathrm{~nm}$ and the hole radius is $115 \mathrm{~nm}$. The calculations are performed within an effective index approximation, in which the $\mathrm{PhC}$ pattern is assumed to be infinitely extended along the $\mathrm{z}$ direction, and embedded in a medium with a fictious refractive index $n=2.66$, which corresponds to the effective refractive index of guided modes propagating in a 170-nm-thick Gallium Arsenide slab at a vacuum wavelength $\lambda=1300 \mathrm{~nm}$. Each single L3 cavity can be tuned by a local change of the refractive index (fig. 3b), resulting in a linear variation of the single-cavity mode frequency (fig. 3c). We now consider three of such cavities arranged in-line, and separated by two lattice periods. Due to the cavity coupling, three supermodes are formed, denoted $a_{-}, a_{0}$ and $a_{+}$(figs. $4(\mathrm{~d}-\mathrm{f})$ ). These modes feature several important characteristics: The mode $a_{0}$ has frequency $\omega_{\mathrm{c}}$ (same as the single cavity) and its field pattern (fig. 4e, $y$-component of the electric field) is anti-symmetric for reflections across the $y z$-plane. The other two modes have frequencies $\omega_{\mathrm{c}} \pm J$, and their field patterns are both symmetric. In the central cavity the field of the mode $a_{0}$ is zero, while the other two modes have their maximum amplitude. Finally, we note that the modes $a_{+}$and $a_{-}$have opposite phases in the lateral cavities. Therefore, according to eq. 7 (and following discussion), the mode $a_{0}$ is expected to have large field variations in the central cavity (and no change of frequency) upon an anti-symmetric perturbation, which in this case can be obtained by detuning the lateral cavities in an equal and opposite way while leaving the central cavity unaltered.

This behaviour is shown in fig. 4. An anti-symmetric perturbation is obtained by increasing the refractive index in the right cavity by an amount $\Delta n$, and decrease it by the same amount in the left cavity (fig. 4a). As expected, the frequencies of all the supermodes (fig. 4b) are constant for small values of $\Delta n$. The mode $a_{0}$ is actually strictly dispersionless for all the values of $\Delta n$. This is because, in this particular three-mode system, 

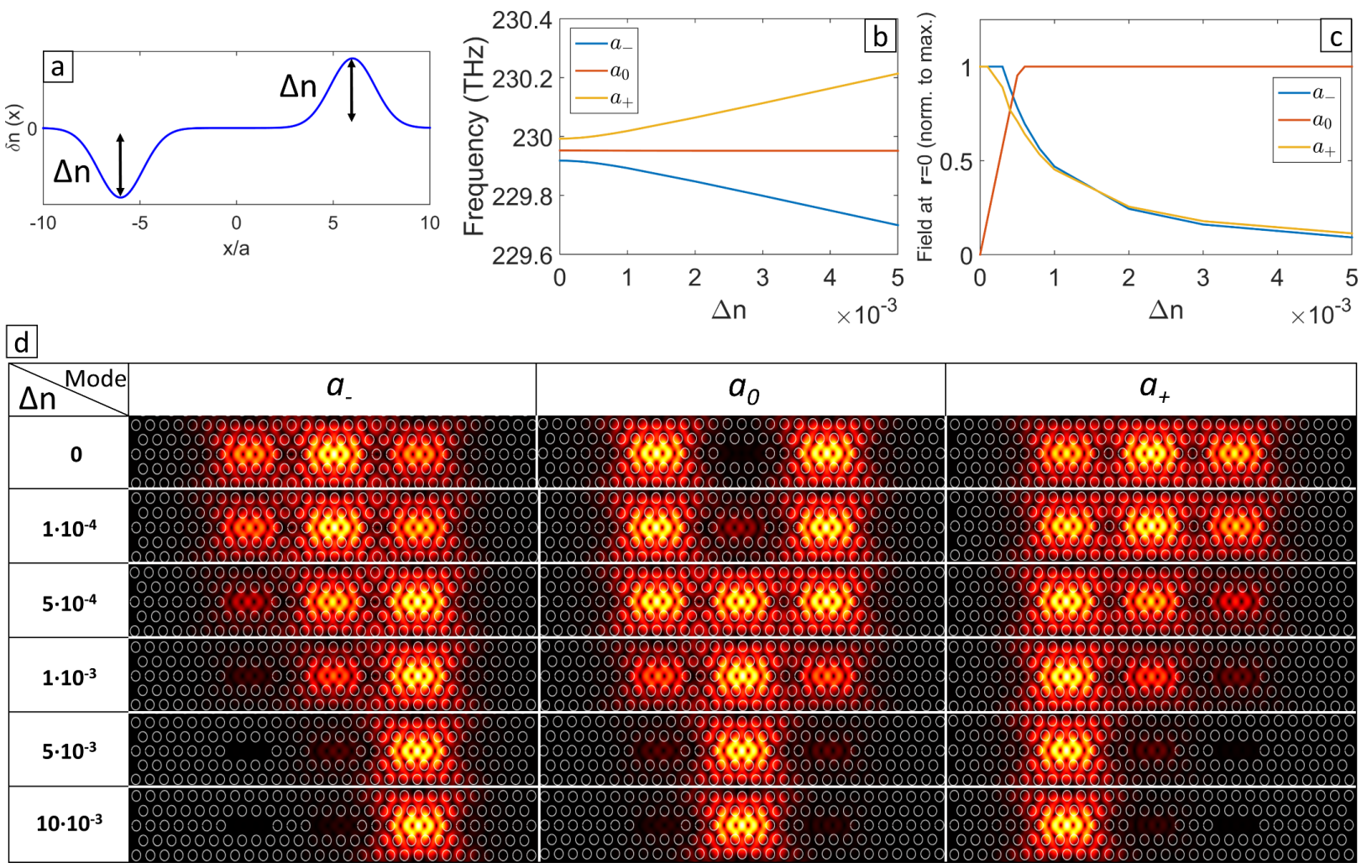

Figure 4. Behaviour of the three-cavity system under an anti-symmetric perturbation. (a) The bulk refractive index is modulated such that the refractive index in the right (left) cavity is increased (decreased) by $\Delta n$. (b) Frequencies of the three supermodes upon the anti-symmetric perturbation versus $\Delta n$. (c) Field amplitudes of the three supermodes at the position $\mathbf{r}=0$, normalized by their maximum, versus $\Delta n$. (d) Evolution of the field amplitudes of the three supermodes for increasing values of $\Delta n$, as indicated in the first column. The colorbar of all the plots in panel $\mathrm{d}$ is the same as in fig. 3 a.
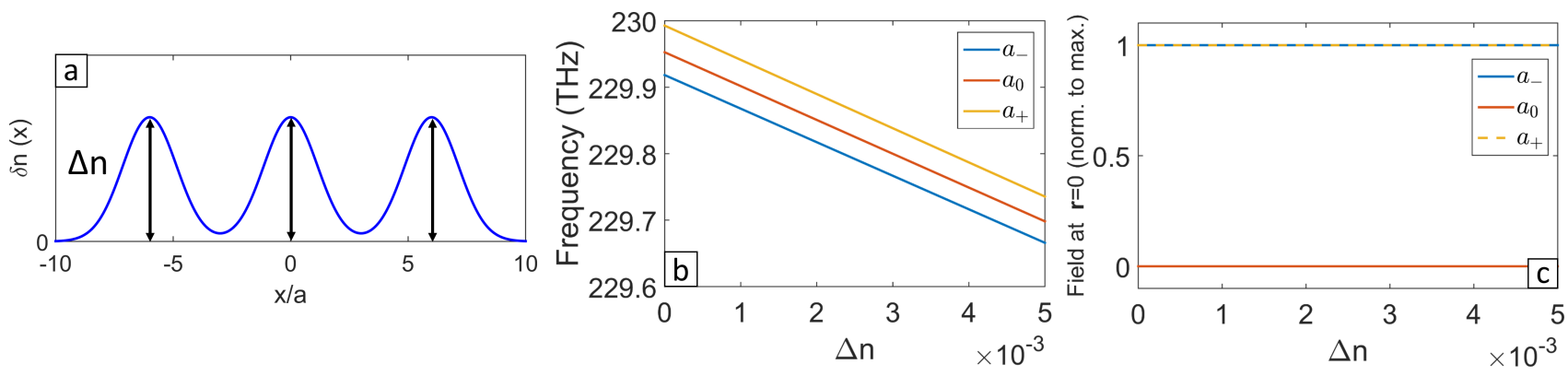

Figure 5. Behaviour of the three-cavity system under a symmetric perturbation. (a) The bulk refractive index is modulated such that the refractive index in each cavity is increased by $\Delta n$. (b) Frequencies of the three supermodes upon the symmetric perturbation versus $\Delta n$. (c) Field amplitudes of the three supermodes at the position $\mathbf{r}=0$, normalized by their maximum, versus $\Delta n$.

the perturbative corrections to the frequency of $a_{0}$ are null at every perturbative order as a result of destructive interference of the terms due to the modes $a_{-}$and $a_{+}$. In fig. $4 \mathrm{~d}$ we show the evolution of the field patterns of the three supermodes for increasing values of $\Delta n$. The electric field of the mode $a_{0}$, initially confined in the lateral cavities, becomes more and more localized to the central cavity as the perturbation is increased. As shown in the plot in fig. 4c (solid red line), a variation of the refractive index of $\Delta n<10^{-3}$ is sufficient to have the maximum of the electric field of the mode $a_{0}$ in the central cavity $(i . e$. , at $\mathbf{r}=0$ ). The other two optical modes 
also show a strong variation of the field pattern: the electric field, initially delocalized over all the three cavities, localizes in one of the two lateral cavities for large $\Delta n$. The variation of the fields of the modes $a_{-}$and $a_{+}$in the central cavity (fig. 4c, solid yellow and blue lines) is however less marked than the one of the mode $a_{0}$ : This is because at the first perturbative order the modes $a_{-}$and $a_{+}$can couple only to the mode $a_{0}^{\dagger}$ which is zero in the central cavity, and therefore the perturbative corrections to the field patterns are of the second or higher order. In striking contrast, when the system is symmetrically perturbed (by, e.g., changing the refractive index in the same way in all the cavities, see fig. 5a), the frequencies of all the supermodes change linearly with $\Delta n$ (fig. 5b), while no variation of the field patterns occur (fig. 5c).

It is interesting to compare the results of fig. 2e and fig. 4c. While for the case of a single cavity a variation of the refractive index of $\Delta n \approx 0.1$ is necessary to change the field of the second mode (fig. 2e, green line) from zero to its maximum value, the refractive index variation required to have the same effect in the three-cavity system is more than two orders of magnitude smaller. This strong difference is the result of the largely reduced frequency spacing obtainable in a coupled-cavity system with respect to a single cavity. The variation of the field amplitude can be made even stronger by reducing the coupling, and thereby the frequency spacing of the supermodes, with practical limits dictated by the quality factor and by fabrication imperfections which may lead to localization.

\section{CONCLUSIONS}

We discussed simple design principles which allow obtaining large variations of the field of an optical cavity, without change of the frequency, upon an external perturbation. In particular we showed that, in a symmetric cavity, the field amplitude of one mode in a particular point $\overline{\mathbf{r}}$ can be highly changed by an anti-symmetric perturbation, if other modes of the cavity have large field amplitudes in $\overline{\mathbf{r}}$ and they are spectrally close to the mode of interest. This last requirement of small frequency spacing between the unperturbed modes indicates that systems of coupled cavities, where the frequency spacing between the supermodes can be controlled by the cavity distances, are promising candidates to achieve large field variations. We demonstrated these design rules in two different photonic structures, namely a single Fabry-Perot cavity and a system composed by three coupled photonic crystal cavities. Both systems show field variations with no change in frequency upon a small external perturbation. However, the perturbation required in the three-cavity system to change the field from zero to its maximum value is more than two order of magnitude smaller than the one required in the single cavity to obtain the same effect. As pointed out by our analysis, this large difference arises from the strongly reduced frequency spacings achievable by using the supermodes of a coupled-cavity system.

\section{ACKNOWLEDGMENTS}

The authors acknowledge useful discussions with R. Johne, R. Schutjens, L. Midolo and M. Gurioli. This work is part of the research programme of the Foundation for Fundamental Research on Matter (FOM), which is financially supported by the Netherlands Organisation for Scientific Research (NWO). E.V. gratefully acknowledges an NWO-Vidi grant for financial support.

\section{REFERENCES}

[1] T. Yoshie, A. Scherer, J. Hendrickson, G. Khitrova, H. Gibbs, G. Rupper, C. Ell, O. Shchekin, and D. Deppe, "Vacuum rabi splitting with a single quantum dot in a photonic crystal nanocavity," Nature 432(7014), pp. 200-203, 2004.

[2] S. Mosor, J. Hendrickson, B. Richards, J. Sweet, G. Khitrova, H. Gibbs, T. Yoshie, A. Scherer, O. Shchekin, and D. Deppe, "Scanning a photonic crystal slab nanocavity by condensation of xenon," Appl. Phys. Lett. 87(14), p. 141105, 2005.

[3] L. Midolo, F. Pagliano, T. Hoang, T. Xia, F. van Otten, L. Li, E. Linfield, M. Lermer, S. Höfling, and A. Fiore, "Spontaneous emission control of single quantum dots by electromechanical tuning of a photonic crystal cavity," Appl. Phys. Lett. 101(9), p. 091106, 2012.

${ }^{\dagger}$ The mutual coupling between the modes $a_{-}$and $a_{+}$is zero at the first perturbative order because of their equal symmetry. 
[4] C.-Y. Jin, R. Johne, M. Y. Swinkels, T. B. Hoang, L. Midolo, P. J. van Veldhoven, and A. Fiore, "Ultrafast non-local control of spontaneous emission," Nat. Nanotechnol. 9(11), pp. 886-890, 2014.

[5] R. Johne, R. Schutjens, S. Fattah poor, C.-Y. Jin, and A. Fiore, "Control of the electromagnetic environment of a quantum emitter by shaping the vacuum field in a coupled-cavity system," Phys. Rev. A 91(6), p. 063807, 2015.

[6] B. Wild, R. Ferrini, R. Houdre, M. Mulot, S. Anand, and C. Smith, "Temperature tuning of the optical properties of planar photonic crystal microcavities," Appl. Phys. Lett. 84(6), pp. 846-848, 2004.

[7] M. A. Dundar, F. Bordas, T. J. Eijkemans, N. Chauvin, A. Y. Silov, R. Notzel, F. Karouta, A. Fiore, and R. W. Van der Heijden, "Lithographic and optical tuning of InGaAsP membrane photonic crystal nanocavities with embedded InAs quantum dots," J. Nanophotonics 3(1), pp. 031765-031765, 2009.

[8] F. Raineri, C. Cojocaru, R. Raj, P. Monnier, A. Levenson, C. Seassal, X. Letartre, and P. Viktorovitch, "Tuning a two-dimensional photonic crystal resonance via optical carrier injection," Opt. Lett. 30(1), pp. 6466, 2005.

[9] A. F. Koenderink, M. Kafesaki, B. C. Buchler, and V. Sandoghdar, "Controlling the resonance of a photonic crystal microcavity by a near-field probe," Phys. Rev. Lett. 95(15), p. 153904, 2005.

[10] F. Intonti, S. Vignolini, F. Riboli, A. Vinattieri, D. S. Wiersma, M. Colocci, L. Balet, C. Monat, C. Zinoni, L. H. Li, et al., "Spectral tuning and near-field imaging of photonic crystal microcavities," Phys. Rev. B 78(4), p. 041401, 2008.

[11] C. J. Chang-Hasnain, "Tunable vcsel," IEEE J. Sel. Top. Quantum Electron. 6(6), pp. 978-987, 2000.

[12] X. Chew, G. Zhou, H. Yu, F. S. Chau, J. Deng, Y. C. Loke, and X. Tang, "An in-plane nano-mechanics approach to achieve reversible resonance control of photonic crystal nanocavities," Opt. Express 18(21), pp. 22232-22244, 2010.

[13] R. Perahia, J. Cohen, S. Meenehan, T. Alegre, and O. Painter, "Electrostatically tunable optomechanical zipper cavity laser," Appl. Phys. Lett. 97(19), pp. Art-No, 2010.

[14] I. W. Frank, P. B. Deotare, M. W. McCutcheon, and M. Lončar, "Programmable photonic crystal nanobeam cavities," Opt. Express 18(8), pp. 8705-8712, 2010.

[15] L. Midolo, P. van Veldhoven, M. Dündar, R. Nötzel, and A. Fiore, "Electromechanical wavelength tuning of double-membrane photonic crystal cavities," Appl. Phys. Lett. 98(21), p. 211120, 2011.

[16] L. Midolo, S. Yoon, F. Pagliano, T. Xia, F. van Otten, M. Lermer, S. Höfling, and A. Fiore, "Electromechanical tuning of vertically-coupled photonic crystal nanobeams," Opt. Express 20(17), pp. 19255-19263, 2012.

[17] M. Petruzzella, T. Xia, F. Pagliano, S. Birindelli, L. Midolo, Z. Zobenica, L. Li, E. Linfield, and A. Fiore, "Fully tuneable, purcell-enhanced solid-state quantum emitters," Appl. Phys. Lett. 107(14), p. 141109, 2015.

[18] J. Reithmaier, G. Sek, A. Löffler, C. Hofmann, S. Kuhn, S. Reitzenstein, L. Keldysh, V. Kulakovskii, T. Reinecke, and A. Forchel, "Strong coupling in a single quantum dot-semiconductor microcavity system," Nature 432(7014), pp. 197-200, 2004.

[19] J. J. Degnan, "Theory of the optimally coupled q-switched laser," IEEE J. Quant. Electron. 25(2), pp. 214$220,1989$.

[20] F. Elste, S. Girvin, and A. Clerk, "Quantum noise interference and backaction cooling in cavity nanomechanics," Phys. Rev, Lett. 102(20), p. 207209, 2009.

[21] M. Cotrufo, A. Fiore, and E. Verhagen, "Coherent atom-phonon interaction through mode field coupling in hybrid optomechanical systems," arXiv preprint arXiv:1610.05153, 2016.

[22] J. D. Joannopoulos, R. D. Meade, and J. N. Winn, Molding the flow of light, Photonic Crystals, 1995.

[23] A. Messiah, Quantum Mechanics, Dover Publications, 1961.

[24] F. Intonti, F. Riboli, N. Caselli, M. Abbarchi, S. Vignolini, D. Wiersma, A. Vinattieri, D. Gerace, L. Balet, L. Li, et al., "Youngs type interference for probing the mode symmetry in photonic structures," Phys. Rev. Lett. 106(14), p. 143901, 2011.

[25] N. Caselli, F. Riboli, F. La China, A. Gerardino, L. Li, E. H. Linfield, F. Pagliano, A. Fiore, F. Intonti, and M. Gurioli, "Tailoring the photon hopping by nearest-neighbor and next-nearest-neighbor interaction in photonic arrays," ACS Photonics 2(5), pp. 565-571, 2015.

[26] R. Konoike, H. Nakagawa, M. Nakadai, T. Asano, Y. Tanaka, and S. Noda, "On-demand transfer of trapped photons on a chip," Science Advances 2(5), p. e1501690, 2016. 